\title{
Post infarction ventricular septal rupture: yet hard to treat disease
}

\author{
Toshiaki Ito ${ }^{1}$
}

Received: 5 January 2017 / Accepted: 9 February 2017 / Published online: 9 March 2017

(C) The Japanese Association for Thoracic Surgery 2017

Acute aortic dissection was once a tough enemy for cardiac surgeons, but in-hospital mortality after surgery for acute type A dissection decreased to less than $10 \%$ recently [1]. However, 30-day and in-hospital mortality after acute post infarction ventricular septal rupture (VSR) remains 30-40\% and do not show apparent tendency to improve, showing yearly fluctuations during last 14 years in Japan (Fig. 1). Latest data during 2014 yet showed $28.6 \%$ of 30-day, and $33.5 \%$ of in-hospital mortality [1]. The annual reports tell us another unfavorable fact that yearly case number of VSR surgery is 250 cases at most in comparison with 15,000 of isolated CABG. Even if one institution has 100 of isolated CABG annually, expected case number of VSR is only 1.7. That means many surgeons do not have enough opportunity to become accustomed to surgery for VSR during their whole carrier.

Historically, infarctectomy and direct patch closure was a standard technique for post infarction VSR. In the early 1990s, David et al. [2] advocated the infarct exclusion technique without infarctectomy. Their $13.6 \%$ of in-hospital mortality was striking, therefore, many surgeons adopted this technique and several modifications of this technique were proposed. In the discussion record of meeting presentation of this paper, Daggett mentioned that "I am unable to decide whether the results that Dr. David has been able to

This is a commentry to the article available at doi:10.1007/ s11748-016-0722-4.

Toshiaki Ito

cvs1@nagoya-1st.jrc.or.jp

1 Department of cardiovascular surgery, Japanese Red Cross Nagoya First Hospital, 3-35 Michishita-cho, Nakamura-ku, Nagoya 453-8511, Japan achieve are a result of the design of the operation or of his surgical skill."

17 years later, Arnaoutakis et al. analyzed 12-year data of STS database from 1999 to 2010 [3]. Overall mortality of 2876 cases of post infarction VSP was $42.9 \%$. In the discussion record of meeting presentation of this paper, David mentioned that they had 91 cases of post-infarction VSR at Toronto General Hospital between 1990 and 2010, and the mortality rate was $40 \%$. Two surgeons including him achieved $20 \%$ of mortality, but other 7 surgeons involved in their series underperformed and the final result became exactly the same with STS average. As Daggett concerned, David's personal expertise and dexterity may have played an important role in achieving marvelous initial result of infarct exclusion technique.

The experience at Toronto General Hospital shows us that one surgeon's experience is not necessarily reproducible even in the same institution in surgery for VSR.

Another problem in evaluating a new technique is a reporting bias. If one reads a paper reporting only one death out of 13 consecutive cases, the new technique proposed on the paper may sound attractive. However, if an expected mortality of each case is $30 \%$, the mathematical probability of the "promising" result of $1 / 13$ mortality is $5.4 \%$. Thus, a "good" result in a small series sometimes could occur only by chance, and tends to be reported.

In summary, there is no solid evidence that one specific surgical method can improve result of post infarction VSR.

In this issue of General Thoracic and Cardiovascular Surgery, Isoda et al. analyzed the result of their sandwich technique for VSR. They reported zero 30-day mortality in 24 consecutive cases, that could unlikely occur only by chance. They concluded that large enough patch according to the size of the defect is necessary to prevent residual leak that negatively affects 1 -year mortality. 
Fig. 1 Surgical results of acute post infarction ventricular septal rupture between 2001 and 2014 in Japan. Columns show annual case number, and line shows in-hospital mortality. Data were picked up from annual reports of Japanese Association for Thoracic Surgery

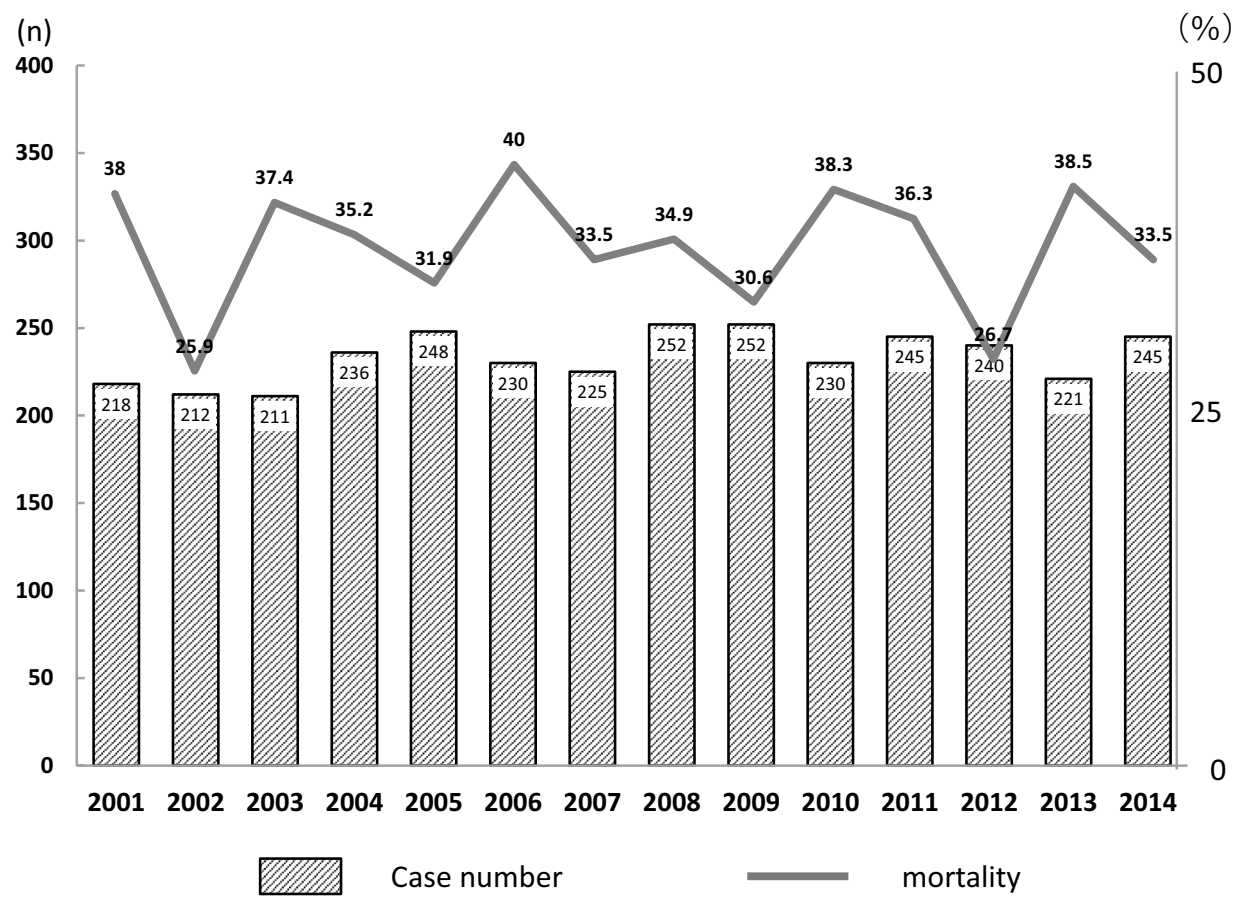

We [4] previously reported geometrical property of patch closure of post infarction VSR using finite element analysis. A large patch that closely contact to endocardium of the left ventricle decreased the stress on the suture line. In addition, increased stiffness of the patch around the septal defect prevented deformity of the patch and reduced stress on the suture line. On the contrary, large defect or too small patch that does not contact to the inner surface of the left ventricle increased stress on the suture line, and may lead to dehiscence of the patch. In sandwich technique, Dacron or Teflon patch soak up surgical glue and become stiff. Two patches may be integrated and act as a plug to obliterate the defect. Thus, a stiffening effect of surgical glue may have decreased stress on the suture-line.

We need a simpler method that is least affected by surgical expertize of each surgeon. Surgical glue may be an answer. Also in left ventricular approach, by gluing felt strips to the epicardium, bleeding from the closure line of the ventricle can be prevented [4]. If sandwich technique is performed as a mirror image through left ventricular approach as Caimmi et al. reported [5], placing larger patch on the left ventricular side becomes easier compared with original right ventricular approach.

The results of last three decades tell us that needle and stitches may not be effective enough to treat post infarction
VSR. I suspect that active use of chemicals as surgical glue or new device as sutureless plug may be necessary to further improve surgical results of post infarction VSR.

\section{Compliance with ethical standards}

Conflict of interest None declared.

\section{References}

1. Masuda M, Okumura M, Doki Y, et al. Thoracic and cardiovascular surgery in Japan during 2014. Annual report by The Japanese Association for Thoracic Surgery. Gen Thorac Cardiovasc Surg. 2016;64:665-97.

2. David TE, Dale L, Sun Z. Postinfarction ventricular septal rupture: repair by endocardial patch with infarct exclusion. J Thorac Cardiovasc Surg. 1995;110:1315-22.

3. Arnaoutakis GJ, Zhao Y, George TJ, et al. Surgical repair of ventricular septal defect after myocardial infarction: outcomes from the society of thoracic surgeons national database. Ann Thorac Surg. 2012;94:436-44.

4. Ito T, Hagiwara H, Maekawa A, et al. Finite element analysis regarding patch size, stiffness, and contact condition to the endocardium in surgery for post infarction ventricular septal rupture. Gen Thorac Cardiovasc Surg. 2013;61:631-39.

5. Caimmi PP, Grossini E, Kapetanakis EI, et al. Double patch repair through a single ventriculotomy for ischemic ventricular septal defects. Ann Thorac Surg. 2010;89:1679-81. 